\title{
Generalised isothermal models with strange equation of state
}

\author{
S. D. Maharaj and S. Thirukkanesh ${ }^{\dagger}$ \\ Astrophysics and Cosmology Research Unit, \\ School of Mathematical Sciences, \\ University of KwaZulu-Natal, \\ Private Bag X54001, \\ Durban 4000, \\ South Africa.
}

\begin{abstract}
We consider the linear equation of state for matter distributions that may be applied to strange stars with quark matter. In our general approach the compact relativistic body allows for anisotropic pressures in the presence of the electromagnetic field. New exact solutions are found to the Einstein-Maxwell system. A particular case is shown to be regular at the stellar centre. In the isotropic limit we regain the general relativistic isothermal universe. We show that the mass corresponds to values obtained previously for quark stars when anisotropy and charge are present.
\end{abstract}

Keywords: Exact solutions; Einstein-Maxwell spacetimes; relativistic stars.

PACS Nos: $04.20 . J b, 04.40 . \mathrm{Nr}, 97.10 . \mathrm{Cv}$

${ }^{\dagger}$ Permanent address: Department of Mathematics, Eastern University, Sri Lanka, Chenkalady, Sri Lanka. 


\section{Introduction}

In an early and seminal treatment the existence of quark matter in a stellar configuration in hydrostatic equilibrium was suggested by Itoh $\underline{1}$. Subsequently the analysis of strange stars consisting of quark matter has been considered in a number of investigations. Strange stars are likely to form in the period of collapse of the core regions of a massive star after a supernova explosion which was pointed out by Cheng et $a l^{2}$. The core of a neutron star or proto-neutron star is a suitable environment for conventional barotropic matter to convert into strange quark matter. Regions of low temperatures and sufficiently high temperatures are required for a first or second order phase transition which results in deconfined quark matter. Another possibility suggested by Cheng and Dai ${ }^{3}$ to explain the formation of a strange star is the accretion of sufficient mass in a rapidly spinning dense star in X-ray binaries which undergoes a phase transition. The behaviour of matter at ultrahigh densities for quark matter is not well understood: in an attempt to study the physics researchers normally restrict their attention to the MIT bag model (see the treatments of Chodos et al $\underline{\underline{4}}$, Farhi and Jaffe $\underline{\underline{5}}$ and Witten $\underline{6})$. The strange matter equation of state is taken to be

$$
p=\frac{1}{3}(\rho-4 B)
$$

where $\rho$ is the energy density, $p$ is the pressure and $B$ is the bag constant. The vacuum pressure $B$ is the bag model equilibrates the pressure and stabilises the system; the constant $B$ determines the quark confinement. The studies of Bombaci $\underline{7}$, Li et al $\underline{\underline{8}} \underline{\underline{9}}, \underline{10}$, Dey et al $\underline{11}$, $\mathrm{Xu}$ et $a l \underline{12}, \underline{13}$, Pons et al $\underline{14}$ and Usov $\underline{15}$ directed at particular compact astronomical objects suggest that these could be strange stars composed of quark matter with equation of state (11).

Mak and Harko $\frac{16}{}$ found an exact general relativistic model of a quark star that admits a conformal Killing vector. This was shown by Komathiraj and Maharaj $\frac{17}{17}$ to be a part of a more general class of exact analytical models in the presence of the electromagnetic field with isotropic pressures. The role of anisotropy was investigated by Lobo $\frac{18}{}$, Mak and Harko $\underline{19}$ and Sharma and Maharaj $\underline{20}$ for strange stars with quark matter with neutral anisotropic distributions. It is our intention to study the Einstein-Maxwell system with a linear equation of state with anisotropic pressures; this treatment would be applicable to a strange stars which are charged and anisotropic which is the most general case. In $\S 2$, we write the EinsteinMaxwell system in an equivalent form using a coordinate transformation. A new exact solution, in terms of simple elementary functions, is given in $\S 3$. In addition, we demonstrate that it is possible to find a particular model which is nonsingular at the stellar origin. The limit of vanishing anisotropy is studied in $\S 4$ and we regain the isothermal universes studied previously. In $\S 5$, we consider the physical features of the new solutions, plot the matter variables for particular parameter values and show that the quark star mass is consistent with earlier treatments. Some concluding remarks are made in $\S 6$. 


\section{Basic equations}

It is our intention to model the interior of a dense realistic star with a general matter distribution. On physical grounds we can take the gravitational field to be static and spherically symmetric. Consequently, we assume that the gravitational field of the stellar interior is represented by the line element

$$
d s^{2}=-e^{2 \nu(r)} d t^{2}+e^{2 \lambda(r)} d r^{2}+r^{2}\left(d \theta^{2}+\sin ^{2} \theta d \phi^{2}\right)
$$

in Schwarzschild coordinates $\left(x^{a}\right)=(t, r, \theta, \phi)$. We consider the general case of a matter distribution with both anisotropy and charge. Therefore we take the energy momentum tensor for the interior to be an anisotropic charged imperfect fluid; this is represented by the form

$$
T_{i j}=\operatorname{diag}\left(-\rho-\frac{1}{2} E^{2}, p_{r}-\frac{1}{2} E^{2}, p_{t}+\frac{1}{2} E^{2}, p_{t}+\frac{1}{2} E^{2}\right),
$$

where $\rho$ is the energy density, $p_{r}$ is the radial pressure, $p_{t}$ is the tangential pressure and $E$ is the electric field intensity. These physical quantities are measured relative to the comoving fluid velocity $u^{i}=e^{-\nu} \delta_{0}^{i}$. The line element (2) and the imperfect matter distribution (3) generate the Einstein field equations; the field equations can be written in the form

$$
\begin{aligned}
\frac{1}{r^{2}}\left[r\left(1-e^{-2 \lambda}\right)\right]^{\prime} & =\rho+\frac{1}{2} E^{2}, \\
-\frac{1}{r^{2}}\left(1-e^{-2 \lambda}\right)+\frac{2 \nu^{\prime}}{r} e^{-2 \lambda} & =p_{r}-\frac{1}{2} E^{2}, \\
e^{-2 \lambda}\left(\nu^{\prime \prime}+\nu^{\prime 2}+\frac{\nu^{\prime}}{r}-\nu^{\prime} \lambda^{\prime}-\frac{\lambda^{\prime}}{r}\right) & =p_{t}+\frac{1}{2} E^{2}, \\
\sigma & =\frac{1}{r^{2}} e^{-\lambda}\left(r^{2} E\right)^{\prime},
\end{aligned}
$$

where primes denote differentiation with respect to $r$ and $\sigma$ is the proper charge density. We are utilising units where the coupling constant $\frac{8 \pi G}{c^{4}}=1$ and the speed of light $c=1$. The Einstein-Maxwell system of equations (44)-(7) describes the gravitational behaviour for an anisotropic charged imperfect fluid. For matter distributions with $p_{r}=p_{t}$ (isotropic pressures) and $E=0$ (no charge) we regain Einstein's equations for an uncharged perfect fluid from (44)-(7).

An equivalent form of the field equations is obtained if we introduce new variables: the independent variable $x$ and new functions $y$ and $Z$. These are given by

$$
x=C r^{2}, \quad Z(x)=e^{-2 \lambda(r)} \text { and } A^{2} y^{2}(x)=e^{2 \nu(r)},
$$

which was earlier used by Durgapal and Bannerji 21 to describe neutron stars. In terms of the new variables the line element (2) becomes

$$
d s^{2}=-A^{2} y^{2} d t^{2}+\frac{1}{4 C x Z} d x^{2}+\frac{x}{C}\left(d \theta^{2}+\sin ^{2} \theta d \phi^{2}\right),
$$


where $A$ and $C$ are arbitrary constants. The transformation (8) simplifies the field equations, and we find that the system (44)-(7) can be written as

$$
\begin{aligned}
\frac{1-Z}{x}-2 \dot{Z} & =\frac{\rho}{C}+\frac{E^{2}}{2 C}, \\
4 Z \frac{\dot{y}}{y}+\frac{Z-1}{x} & =\frac{p_{r}}{C}-\frac{E^{2}}{2 C}, \\
4 x Z \frac{\ddot{y}}{y}+(4 Z+2 x \dot{Z}) \frac{\dot{y}}{y}+\dot{Z} & =\frac{p_{t}}{C}+\frac{E^{2}}{2 C}, \\
\frac{\sigma^{2}}{C} & =\frac{4 Z}{x}(x \dot{E}+E)^{2},
\end{aligned}
$$

where dots denote differentiation with respect to the variable $x$.

The definition

$$
m(r)=\frac{1}{2} \int_{0}^{r} \omega^{2} \rho(\omega) d \omega
$$

represents the mass contained within a radius $r$ which is a useful physical quantity. The mass function (14) has the form

$$
m(x)=\frac{1}{4 C^{3 / 2}} \int_{0}^{x} \sqrt{w} \rho(w) d w,
$$

in terms of the new variables introduced in (8).

On physical grounds we expect that the matter distribution for realitic stellar matter should satisfy a barotropic equation of state $p_{r}=p_{r}(\rho)$. For the investigations in this paper we assume the particular equation of state

$$
p_{r}=\alpha \rho-\beta,
$$

where $\alpha$ and $\beta$ are constants. This is a simple linear relationship with desirable physical features and contains models investigated previously. Now it is possible to rewrite (10)-(13) as the system

$$
\begin{aligned}
\frac{\rho}{C} & =\frac{1-Z}{x}-2 \dot{Z}-\frac{E^{2}}{2 C} \\
p_{r} & =\alpha \rho-\beta \\
p_{t} & =p_{r}+\Delta \\
\Delta & =4 C x Z \frac{\ddot{y}}{y}+2 C\left[x \dot{Z}+\frac{4 Z}{(1+\alpha)}\right] \frac{\dot{y}}{y}+\frac{(1+5 \alpha)}{(1+\alpha)} C \dot{Z}-\frac{C(1-Z)}{x}+\frac{2 \beta}{(1+\alpha)}, \\
\frac{E^{2}}{2 C} & =\frac{1-Z}{x}-\frac{1}{(1+\alpha)}\left[2 \alpha \dot{Z}+4 Z \frac{\dot{y}}{y}+\frac{\beta}{C}\right], \\
\frac{\sigma^{2}}{C} & =4 \frac{Z}{x}(x \dot{E}+E)^{2},
\end{aligned}
$$

where the quantity $\Delta=p_{t}-p_{r}$ is defined as the measure of anisotropy. The Einstein-Maxwell equations as expressed in (17)-(22) is a system of six nonlinear equations in terms of eight variables $\left(\rho, p_{r}, p_{t}, \Delta, E, \sigma, y, Z\right)$. The system (17)-(22) is under-determined so that there are different ways in which we can proceed with the integration process. Here we show that it 
is possible to specify two of the quantities and generate an ordinary differential equation in only one dependent variable in the integration process. This helps to produce a particular exact model.

\section{New solutions}

In this paper, we choose physically reasonable forms for the gravitational potential $Z$ and electric field intensity $E$ and then integrate the system (17)-(22) to generate exact models. We make the specific choices

$$
\begin{aligned}
Z & =\frac{1}{a+b x^{n}} \\
\frac{E^{2}}{C} & =\frac{2 k(d+2 x)}{a+b x^{n}}
\end{aligned}
$$

where $a, b, d, n$ and $k$ are real constants. The potential $Z$ is regular at the origin and continuous in the stellar interior for a wide range of values for the parameters $a, b$ and $n$. The electric field intensity $E$ is a bounded and decreasing function from the origin to the surface of the sphere. Therefore the forms chosen in (23)-(24) are physically acceptable. These specific choices for $Z$ and $E$ simplify the integration process. Equation (21) can be written as

$$
\frac{\dot{y}}{y}=\frac{(a-1)(1+\alpha)}{4 x}+\frac{\alpha}{2} \frac{b n x^{n-1}}{\left(a+b x^{n}\right)}+\frac{(1+\alpha) b}{4} x^{n-1}-\frac{\beta}{4 C}\left(a+b x^{n}\right)-\frac{(1+\alpha) k}{4}(d+2 x)
$$

where we have used (23) and (24). This has the advantage of being a first order linear equation in the gravitational potential $y$.

Equation (25) can be integrated in closed form to give

$$
y=D x^{\frac{(a-1)(1+\alpha)}{4}}\left(a+b x^{n}\right)^{\frac{\alpha}{2}} \exp [F(x)],
$$

where we have defined

$$
F(x)=-\frac{\beta x}{4 C}\left[a+\frac{b x^{n}}{n+1}\right]+\frac{(1+\alpha)}{4}\left[\frac{b x^{n}}{n}-k\left(d x+x^{2}\right)\right]
$$

and $D$ is a constant of integration. Now from (23), (24) and (26) we can generate an exact 
model for the system (17)-(22) as follows

$$
\begin{aligned}
& e^{2 \lambda}=a+b x^{n}, \\
& e^{2 \nu}=A^{2} D^{2} x^{\frac{(a-1)(1+\alpha)}{2}}\left(a+b x^{n}\right)^{\alpha} \exp [2 F(x)], \\
& \frac{\rho}{C}=\frac{(a-1)+b x^{n}}{x\left(a+b x^{n}\right)}+\frac{2 b n x^{n-1}}{\left(a+b x^{n}\right)^{2}}-\frac{k(d+2 x)}{\left(a+b x^{n}\right)} \\
& p_{r}=\alpha \rho-\beta, \\
& p_{t}=p_{r}+\Delta, \\
& \Delta= \\
& \frac{1}{4}\left\{\frac{4 C\left(1-a-b x^{n}\right)}{x\left(a+b x^{n}\right)}-\frac{4 b C n(1+5 \alpha) x^{n-1}}{(1+\alpha)\left(a+b x^{n}\right)^{2}}+\frac{8 \beta}{(1+\alpha)}-\frac{2\left[4 a-b x^{n}(n(1+\alpha)-4)\right]^{2}}{(1+\alpha) x\left(a+b x^{n}\right)^{3}} \times\right. \\
& \left(a^{2}(\beta x-C(1+\alpha))+b x^{n}\left(C\left[(1+\alpha)\left(1-b x^{n}+d k x+2 k x^{2}\right)-2 n \alpha\right]+b \beta x^{n+1}\right)\right. \\
& \left.+a\left[C(1+\alpha)\left(1+d k x+2 k x^{2}-2 b x^{n}\right)+2 b \beta x^{n+1}\right]\right)+\frac{C x}{\left(a+b x^{n}\right)}\left[\frac{1}{x^{2}}((a-1)(1+\alpha) \times\right. \\
& \left.\left(\frac{4 b n \alpha x^{n}}{\left(a+b x^{n}\right)}+(a(1+\alpha)-5-\alpha)\right)+\frac{4 b n \alpha x^{n}\left(2 a(n-1)+(n \alpha-2) b x^{n}\right)}{\left(a+b x^{n}\right)^{2}}\right) \\
& -\frac{2\left[a(1+\alpha)\left(a-1+b x^{n}\right)+b x^{n}(2 n \alpha-(1+\alpha))\right]}{C x^{2}\left(a+b x^{n}\right)}\left(C(1+\alpha)\left(k\left(d x+2 x^{2}\right)-b x^{n}\right)\right) \\
& \left.+\beta x\left(a+b x^{n}\right)\right)+4\left((1+\alpha)\left(b(n-1) x^{n-2}-2 k\right)-\frac{b n \beta x^{n-1}}{C}+\right. \\
& \left.\left.\left.\frac{\left[C(1+\alpha)\left(k(d+2 x)-b x^{n-1}\right)+\beta\left(a+b x^{n}\right)\right]^{2}}{4 C^{2}}\right)\right]\right\} \\
& \frac{E^{2}}{C}=\frac{2 k(d+2 x)}{a+b x^{n}} .
\end{aligned}
$$

The equations (27)-(33) represent an exact solution to the Einstein-Maxwell system (17)-(22) for a charged imperfect fluid with the linear equation of state $p_{r}=\alpha \rho-\beta$. Exact solutions with the equation of state $p_{r}=\alpha \rho-\beta$ have been used to model compact objects such as strange stars, as shown by Sharma and Maharaj $\underline{20}$, and dark energy stars which are stable, as demonstrated by Lobo $\stackrel{18}{ }$. The exact solution (27)-(33) may be regarded as a generalisation of an isothermal universe model as we indicate in $\S 4$.

The solution (27)-(33) admits singularities at the stellar centre in general. The singularity may be avoided for particular parameter values. If we set $a=1$ and $n=1$ then we generate the line element

$$
d s^{2}=-A^{2} D^{2}(1+b x)^{\alpha} \exp [2 F(x)] d t^{2}+(1+b x) d r^{2}+r^{2}\left(d \theta^{2}+\sin ^{2} \theta d \phi^{2}\right),
$$

where $F(x)=-\frac{\beta x}{8 C}(2+b x)+\frac{(1+\alpha)}{4}\left[b x-k\left(d x+x^{2}\right)\right]$, with energy density

$$
\frac{\rho}{C}=\frac{b(3+b x)}{(1+b x)^{2}}-\frac{k(d+2 x)}{(1+b x)} .
$$

When $k=0$ then the mass function (15) has the form

$$
m(x)=\frac{b x^{3 / 2}}{2 C^{3 / 2}(1+b x)} .
$$


The expression of the mass function given in (35) represents an energy density which is monotonically decreasing in the interior of the uncharged sphere and has a finite value at the centre $x=0$. This is physically reasonable and similar mass profiles appear in the treatments of general relativistic equilibrium configurations of Matese and Whitman ${ }^{22}$, neutron star models of Finch and Skea 23 , anisotropic stellar solutions of Mak and Harko 24 , and the dark energy stars of Lobo $\stackrel{18}{ }$. The gravitational potentials for charged imperfect fluid solution corresponding to the line element (34) are nonsingular at the origin. The energy density $\rho$, the measure of anisotropy $\Delta$, and associated quantities $p_{r}$ and $p_{t}$ are also regular at the centre for our choice of $E$. Note that the quantity $\Delta$ vanishes at the centre and is a continuous function in the stellar interior. Hence the model (27)-(33) admits a particular case corresponding to the parameter values $a=1$ and $n=1$ which is regular and well behaved at the origin.

\section{Isotropic models}

It is possible to consider the special case of isotropic pressures with $p_{r}=p_{t}$ in the uncharged limit for neutral matter. When $k=0(E=0)$ equation (32) becomes

$$
\begin{aligned}
& \Delta= \\
& \frac{1}{4}\left\{\frac{4 C\left(1-a-b x^{n}\right)}{x\left(a+b x^{n}\right)}-\frac{4 b C n(1+5 \alpha) x^{n-1}}{(1+\alpha)\left(a+b x^{n}\right)}+\frac{8 \beta}{(1+\alpha)}-\frac{2\left[4 a-(n(1+\alpha)-4) b x^{n}\right]}{(1+\alpha) x\left(a+b x^{n}\right)^{3}}\left[a^{2} \times\right.\right. \\
& (\beta x-C(1+\alpha))-b x^{n}\left(C\left((1+\alpha)\left(b x^{n}-1\right)+2 n \alpha\right)-b \beta x^{n+1}\right)+a\left(C(1+\alpha)\left(1-2 b x^{2}\right)\right. \\
& \left.\left.+2 b \beta x^{n+1}\right)\right]+\frac{C}{x\left(a+b x^{n}\right)}\left[4 b(n-1)(1+\alpha) x^{n}+(a-1)(1+\alpha)(a(1+\alpha)-5-\alpha)\right. \\
& +\frac{4(a-1) b n \alpha(1+\alpha) x^{n}}{\left(a+b x^{n}\right)}-\frac{4 b n \beta x^{n+1}}{C}+\frac{4 b n \alpha\left[2 a(n-1)+b(n \alpha-2) x^{n}\right] x^{n}}{\left(a+b x^{n}\right)^{2}} \\
& +\frac{\left[a \beta x-b(C(1+\alpha)-\beta x) x^{n}\right]^{2}}{C^{2}}+\frac{2}{C\left(a+b x^{n}\right)}\left(a(1+\alpha)\left(a-1+b x^{n}\right)\right. \\
& \left.\left.\left.+(2 n \alpha-(1+\alpha)) b x^{n}\right)\left((C(1+\alpha)-\beta x) b x^{n}-a \beta x\right)\right]\right\} .
\end{aligned}
$$

Equation (36) shows that the model remains anisotropic even for the uncharged case in general. However for particular parameter values we can show that $\Delta=0$ in the relevant limit in the general solution (27)-(33). If we set $b=0$ and $\beta=0$ then (36) becomes

$$
\Delta=\frac{C(a-1)}{4 a x}\left\{a(1+\alpha)^{2}-\left[4 \alpha+(1+\alpha)^{2}\right]\right\} .
$$

From the above equation we easily observe that when $a=1$ and $a=1+\frac{4 \alpha}{(1+\alpha)^{2}}$ the measure of anisotropy $\Delta$ vanishes. When $a=1$ we note from (29) that $\rho=0$ since $b=k=0$. Consequently we cannot regain an isotropic model when $a=1$; to avoid vanishing energy densities we must have $a \neq 1$ when $\Delta=0$. When $a=1+\frac{4 \alpha}{(1+\alpha)^{2}}$ we obtain the expressions

$$
\begin{aligned}
d s^{2} & =-B r^{\frac{4 \alpha}{1+\alpha}} d t^{2}+\left[1+\frac{4 \alpha}{(1+\alpha)^{2}}\right] d r^{2}+r^{2}\left(d \theta^{2}+\sin ^{2} \theta d \phi^{2}\right), \\
\rho & =\frac{4 \alpha}{\left[4 \alpha+(1+\alpha)^{2}\right] r^{2}}, \quad p_{r}\left(=p_{t}\right)=\alpha \rho,
\end{aligned}
$$


where we have set $A^{2} D^{2} a^{\alpha}=B$ and $C=1$.

The above solution was obtained by Saslaw et $a l^{25}$ in their investigation of general relativistic isothermal universes. Since $\rho \propto r^{-2}$ we may interpret (37) as a relativistic cosmological metric since there is an analogy that can be made with the well known Newtonian solution as pointed out by Chandrasekhar 26 . In the Newtonian case the density $\rho$ is finite in the core regions and decreases according to $r^{-2}$ in the rest of the model. The total mass and region of the isothermal model are infinite. Therefore the line element (37) may be interpreted as a relativistic inhomogeneous universe where the nonzero pressure balances gravity. Saslaw et $a l^{25}$ point out that (37) may be viewed as the asymptotic state of the Einstein-de Sitter cosmological model, in an expansion-free state as $t \rightarrow \infty$, where the hierarchial distribution of matter has clustered over large scales. As $t \rightarrow \infty$ the Einstein-de Sitter model tends to the line element (37) and the universe evolves into an isothermal static sphere given by the exact solution (37) with equation of state $p_{r}\left(=p_{t}\right)=\alpha \rho$. To move from the pressure-free Einstein-de Sitter model to the static isothermal metric requires a phase transition for condensation through clustering of galaxies. It is interesting to observe that Chaisi and Maharaj 27 and Maharaj and Chaisi 28 have obtained generalised anisotropic static isothermal spheres by utilising a known isotropic metric to produce a new anisotropic solution of the Einstein field equations. Govender and Govinder ${ }^{29}$ have found simple nonstatic generalisations of isothermal universes which describe an isothermal sphere of galaxies in quasi-hydrostatic equilibrium with heat dissipation driving the system to equilibrium. Hence the imperfect relativistic fluid, in the presence of electromagnetic field, with the linear equation of state $p_{r}=\alpha \rho-\beta$, is a generalisation of the conventional isothermal model with a clear physical basis.

\section{Physical analysis}

We observe that the exact solution (27)-(33) may be singular at the origin in general. The solution should be used to describe the gravitational field of the envelope in the outer regions of a quark star or a dark energy star with equation of state $p_{r}=\alpha \rho-\beta$. To avoid the singularity at the centre another solution is required to model the stellar core. Examples of core-envelope models in general relativity are provided by Thomas et al $\underline{30}$, Tikekar and Thomas 31 and Paul and Tikekar 32 . We observe that if $a=1$ then the imperfect charged solution (27)-(33) admits the line element

$$
d s^{2}=-A^{2} D^{2}\left(1+b x^{n}\right)^{\alpha} \exp [2 F(x)] d t^{2}+\left(1+b x^{n}\right) d r^{2}+r^{2}\left(d \theta^{2}+\sin ^{2} \theta d \phi^{2}\right),
$$

where $F(x)=-\frac{\beta x}{4 C}\left[1+\frac{b x^{n}}{n+1}\right]+\frac{(1+\alpha)}{4}\left[\frac{b x^{n}}{n}-k\left(d x+x^{2}\right)\right]$. It is clear from (39) that the gravitational potentials are nonsingular at the origin for all values of $n$. If $n=1$ then there is no singularity in the energy density $\rho$ and the model is nonsingular throughout the stellar interior. However if $n \neq 1$ then the gravitational potential potentials (39) may continue to be well behaved but singularities may appear in the matter variables at the origin $r=0$.

By considering a particular example we can demonstrate graphically that the matter variables are well behaved outside the origin. Figures 1 , 5 represent the energy density, the radial 
pressure, the tangential pressure, the electric field intensity and the measure of anisotropy, respectively. Note that solid lines represent uncharged matter $(E=0)$ and dashed lines include the effect of charged matter $E \neq 0$. To plot the graphs we choose the parameters $n=2, a=1, b=40, k=2, d=1, \alpha=\frac{1}{3}$ and $\beta=0.3569$, and the stellar boundary is set at $r=1$. From Figures 112 we see that both the energy density $\rho$ and the radial pressure $p_{r}$ are continuous throughout the interior, increasing from the centre to $r=0.32$ and then decreasing. Note that the radial pressure is zero at the boundary $r=1$ for the uncharged case $E=0$. We observe from Figure 3 that the tangential press $p_{t}$ is continuous and well behaved in the interior regions. From Figure 4 we observe that the electric field intensity $E$ is decreasing smoothly throughout the stellar interior. We can observe from Figure 5 that the measure of anisotropy is continuous throughout the stellar interior. The behaviour of $\Delta$ outside the centre is likely to correspond to physically realistic matter in the presence of the electromagnetic field. Figure 5 has a profile similar to the anisotropic boson stars studied by Dev and Gleiser $\underline{33}$ and the compact anisotropic relativistic spheres of Chaisi and Maharaj $\underline{34}$. From the figures we can see that the effect of electric field intensity $E$ is to produce lower values for $\rho, p_{r}, p_{t}$ and $\Delta$.

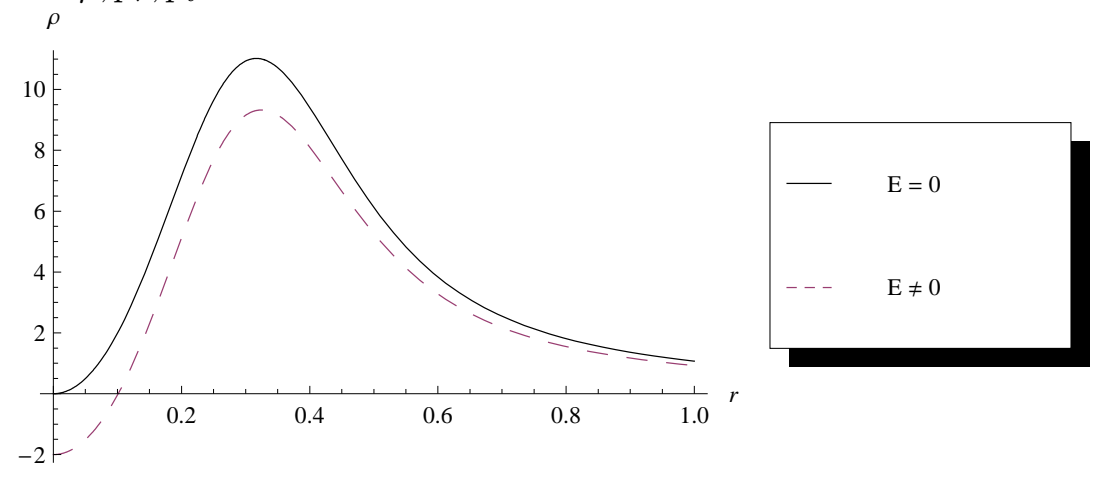

Figure 1: Energy density.

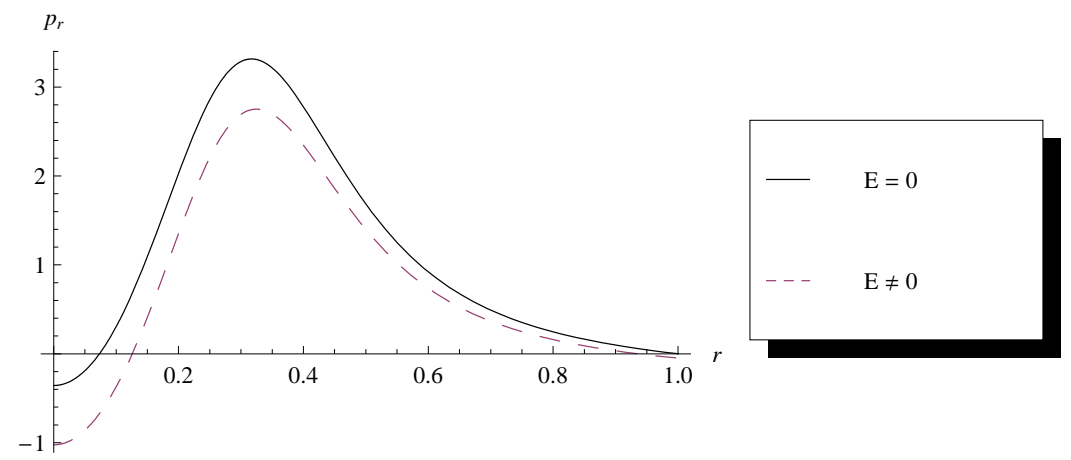

Figure 2: Radial pressure. 


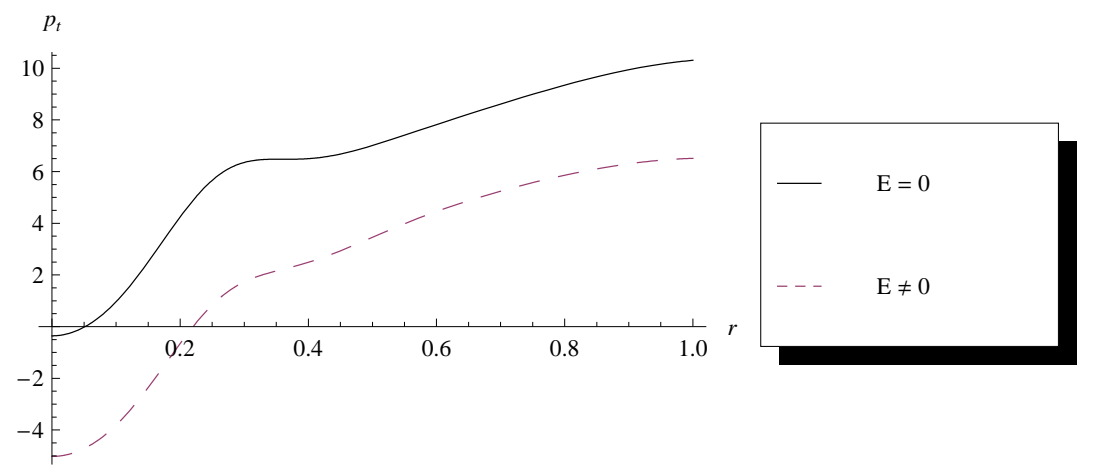

Figure 3: Tangential pressure.

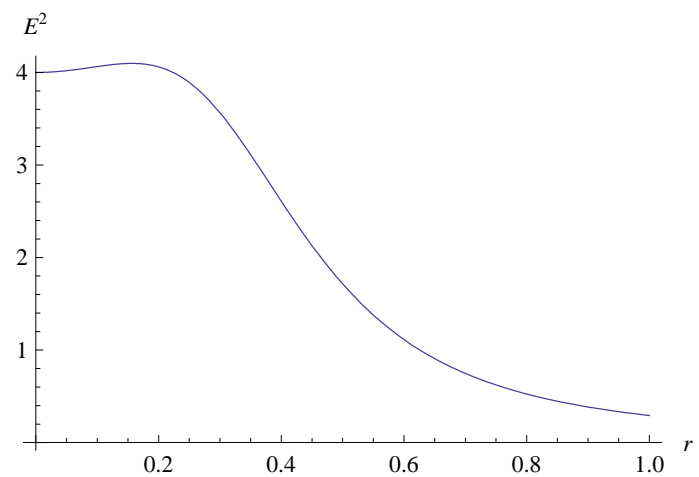

Figure 4: Electric field intensity.

We now show that the solutions generated in this paper can be used to describe realistic compact objects for the case $n=1$ as an example from $\S 3$. In our model, when $n=1$, the parameters $b$ has the dimension of length ${ }^{-2}, k$ has the dimension of length ${ }^{-4}$ and $d$ has the dimension of length ${ }^{2}$. For simplicity, we introduce the transformations

$$
\tilde{b}=b R^{2}, \tilde{k}=k R^{4}, \tilde{d}=d R^{-2},
$$

where $R$ is a parameter which has the dimension of a length. Under these transformations the energy density becomes

$$
\rho=\frac{1}{R^{2}}\left[\frac{\tilde{b}}{1+\tilde{b} y}+\frac{2 \tilde{b}}{(1+\tilde{b} y)^{2}}-\frac{\tilde{k}(\tilde{d}+2 y)}{1+\tilde{b} y}\right],
$$

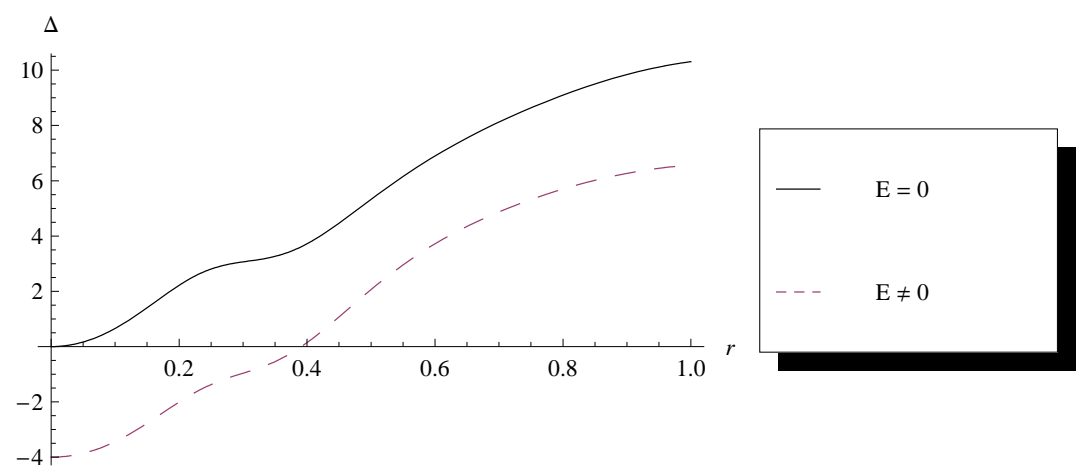

Figure 5: Measure of anisotropy. 
where we have set $C=1$ and $y=\frac{r^{2}}{R^{2}}$. Then the mass contained within a radius $s$ has the form

$$
M=\frac{1}{2}\left\{\frac{\tilde{b} \frac{s^{3}}{R^{2}}}{1+\tilde{b} \frac{s^{2}}{R^{2}}} \frac{2 \tilde{k}}{3 \tilde{b}} \frac{s^{3}}{R^{2}}+\tilde{k}(2-\tilde{b} \tilde{d})\left[\frac{s}{\tilde{b}^{2}}-\frac{R}{\tilde{b}^{5 / 2}} \arctan \left[\sqrt{\tilde{b}} \frac{s}{R}\right]\right]\right\} .
$$

For simplicity we set $\tilde{b} \tilde{d}=2$ so that these expressions reduce to

$$
\begin{aligned}
\rho & =\frac{1}{R^{2}}\left[\frac{\tilde{b}}{1+\tilde{b} y}+\frac{2 \tilde{b}}{(1+\tilde{b} y)^{2}}-\frac{2 \tilde{k}}{\tilde{b}}\right], \\
M & =\frac{1}{2}\left[\frac{\tilde{b}}{1+\tilde{b} \frac{s^{2}}{R^{2}}}-\frac{2 \tilde{k}}{3 \tilde{b}}\right] \frac{s^{3}}{R^{2}},
\end{aligned}
$$

which are simple forms. It is now easy to calculate the density and mass for particular parameter values from (40) and (42). For example, when $s=7.07 \mathrm{~km}, R=1 \mathrm{~km}, \tilde{b}=0.03$ and $\tilde{k}=0.00045$, we obtain the mass $M_{E=0}=1.436 M_{\odot}$ for uncharged matter $M_{E \neq 0}=0.240 M_{\odot}$ for charged matter. Note that the value of the mass for uncharged matter is consistent with the strange star models previously found by Sharma and Maharaj $\underline{20}$ and Dey et al $\underline{11}$. We have shown that the inclusion of the electromagnetic field affects the value for the mass $M$. It is also possible to to relate our results to other treatments. If we set $s=9.46 \mathrm{~km}, R=1 \mathrm{~km}$, $\tilde{b}=0.35$ and $\tilde{k}=0.00045$, then we obtain the mass $M_{E=0}=3.103 M_{\odot}$ for uncharged matter and $M_{E \neq 0}=2.858 M_{\odot}$ for charged matter. These values for the mass are similar to charged quark stellar models generated by Mak and Harko $\underline{16}$ which describe a unique static charged configuration of quark matter admitting a one-parameter group of conformal symmetries. We have shown that the presence of anisotropy and charge in the matter distribution yields masses which are consistent with other investigations.

\section{Discussion}

We have investigated the Einstein-Maxwell system of field equations with a strange matter equation of state for anisotropic matter distributions when the electromagnetic field is present. A new class of exact solutions was found to this system of nonlinear equations. A particular case is nonsingular at the centre of the star and the gravitational potentials and matter variables are well behaved. When the anisotropy vanishes we regain the general relativistic isothermal universe of Saslaw $\underline{25}$. This is an extension of the conventional Newtonian isothermal universe with density $\rho \propto r^{-2}$. The plots of the matter variables indicate that the solution may be used to model quark stars, at least in the envelop if singularities are present at the origin. We calculated the mass in a special case $(a=1, n=1)$ and showed that this value is consistent with strange matter distributions of Dey et al $\underline{11}$, Mak and Harko $\underline{16}$ and Sharma and Maharaj ${ }^{20}$. Ours is a particular solution of the Einstein-Maxwell system with the nice feature of containing the isothermal universe in the isotropic limit. A more comprehensive study of other possible solutions admitted, with the strange matter equation of state, is likely to produce other interesting results. 
We now comment on some physical aspects of the solutions found which are of interest. Firstly, there are a number of free parameters in the solution which may be determined by imposing boundary conditions. The solutions may be connected to the Reissner-Nordstrom exterior spacetime

$$
d s^{2}=-\left(1-\frac{2 M}{r}+\frac{Q^{2}}{r^{2}}\right) d t^{2}+\left(1-\frac{2 M}{r}+\frac{Q^{2}}{r^{2}}\right)^{-1} d r^{2}+r^{2}\left(d \theta^{2}+\sin ^{2} \theta d \phi^{2}\right)
$$

with the interior spacetime (39) across the boundary $r=s$ where $M$ and $Q$ represent the total mass and the charge of the star, respectively. This gives the conditions

$$
\begin{aligned}
1-\frac{2 M}{s}+\frac{Q^{2}}{s^{2}} & =A^{2} D^{2}\left\{\left[1+b\left(C s^{2}\right)^{n}\right]^{\alpha} \exp \left[2 F\left(C s^{2}\right)\right]\right\} \\
\left(1-\frac{2 M}{s}+\frac{Q^{2}}{s^{2}}\right)^{-1} & =1+b\left(C s^{2}\right)^{n} .
\end{aligned}
$$

Therefore the continuity of the metric coefficients across the boundary $r=s$ is maintained. The number of free parameters $(A, C, D, b$ and $n)$ ensures that these necessary conditions are satisfied. Secondly, from the graphs generated for the energy density and the pressure we observe that these quantities are negative in regions close to the centre. We believe that this feature arises because of the strange equation of state that we have imposed on the model. To avoid negative matter variables we would need to use another solution to describe the core with our solution serving as the envelope. This is the situation that arises in the gravastar model of Mazur and Mottola where the singular core is replaced by a de Sitter condensate through a phase transition near the location of the event horizon. This idea has been utilised by Lobo $\frac{18}{}$ to demonstrate that dark energy stars are stable. Thirdly, the example of this paper and other exact solutions found for the Einstein-Maxwell system suggests that the negativity of the energy density and the pressure close to the centre may be a generic feature of charged stellar models. It appears that the existence of the electric field always makes the energy density negative close to the centre of the sphere. This suggests the conjecture that a charged fluid cannot satisfy the energy conditions near the stellar centre is possible. This will be the topic of a separate investigation.

\section{Acknowledgements}

We are grateful to the referee for insightful comments relating to the physical properties of the models. ST thanks the National Research Foundation and the University of KwaZuluNatal for financial support, and is grateful to Eastern University, Sri Lanka for study leave. SDM acknowledges that this work is based upon research supported by the South African Research Chair Initiative of the Department of Science and Technology and the National Research Foundation. 


\section{References}

[1] N Itoh, Progr. Theor. Phys. 44, 291 (1970)

[2] K S Cheng, Z G Dai and T Lu T, Int. J. Mod. Phys. D 7, 139 (1998)

[3] K S Cheng and Z G Dai, Phys. Rev. Lett. 77, 1210 (1996)

[4] A Chodos, R L Jaffe, K Johnson, C B Thorn and V F Weisskopf, Phys. Rev. D 9, 3471 (1974)

[5] E Farhi and R L Jaffe, Phys. Rev. D 30, 2379 (1984)

[6] E Witten, Phys. Rev. D 30, 272 (1984)

[7] I Bombaci, Phys. Rev. C 55, 1587 (1997)

[8] X -D Li, Z -G Dai and Z -R Wang, Astron. Astrophys. 303, L1 (1995)

[9] X -D Li, I Bombaci, M Dey, J Dey and E P J van den Heuvel, Phys. Rev. Lett. 83, 3776 (1999)

[10] X -D Li, S Ray, J Dey, M Dey and I Bombaci, Astrophys. J. 527, L51 (1999)

[11] M Dey, I Bombaci, J Dey, S Ray and B C Samanta, Phys. Lett. B 438, 123 (1998)

[12] R X Xu, G J Qiao and B Zhang, Astrophys. J 522, L109 (1999)

[13] R X Xu, X B Xu and X J Wu, Chin. Phys. Lett. 18, 837 (2001)

[14] J A Pons, F M Walter, J M Lattimer, M Prakash, R Neuhäuser and A Penghui, Astrophys. J. 564, 981 (2002)

[15] V V Usov, Phys. Rev. D 70, 067301 (2004)

[16] M K Mak and T Harko, Int. J. Mod. Phys. D 13, 149 (2004)

[17] K Komathiraj and S D Maharaj, Int. J. Mod. Phys. D 16, 1803 (2007)

[18] F S N Lobo, Class. Quantum Grav. 23, 1525 (2006)

[19] M K Mak and T Harko, Chin. J. Astron. Astrophys. 2, 248 (2002)

[20] R Sharma R and S D Maharaj, Mon. Not. R. Astron. Soc. 375, 1265 (2007)

[21] M C Durgapal and R Bannerji, Phys. Rev. D 27, 328 (1983)

[22] J J Matese and P G Whitman, Gen. Relativ. Gravit. 37, 1270 (1980)

[23] M R Finch and J E F Skea, Class. Quantum Grav. 6, 467 (1989)

[24] M K Mak and T Harko, Proc. Roy. Soc. Lond. A 459, 393 (2003) 
[25] W C Saslaw, S D Maharaj and N Dadhich, Astrophys. J. 471, 571 (1996)

[26] S Chandrasekhar, Introduction to the Study of Stellar Structure (University of Chicago Press, Chicago, 1939)

[27] M Chaisi and S D Maharaj, Pramana -J. Phys. 66, 313 (2006)

[28] S D Maharaj and M Chaisi, Math. Meth. Appl. Sci. 29, 67 (2006)

[29] M Govender and K S Govinder, Int. J. Theor. Phys. 43, 2253 (2004)

[30] V O Thomas, B S Ratanpal and P C Vinodkumar, Int. J. Mod. Phys. D 14, 85 (2005)

[31] R Tikekar and V O Thomas, Pramana -J. Phys. 50, 95 (1998)

[32] B C Paul and R Tikekar, Grav. Cosmol. 11, 244 (2005)

[33] K Dev and M Gleiser, Gen. Relativ. Gravit. 34, 1793 (2005)

[34] M Chaisi and S D Maharaj, Gen Relativ. Gravit. 37, 1177 (2005) 